Vietnam Journal of Mechanics, VAST, Vol.41, No. 2 (2019), pp. 157-170

DOI: https://doi.org/10.15625/0866-7136/13092

\title{
RESONANT AND ANTIRESONANT FREQUENCIES OF MULTIPLE CRACKED BAR
}

\author{
P. T. B. Lien ${ }^{1}$, N. T. Khiem ${ }^{2, *}$ \\ ${ }^{1}$ University of Transport and Communications, Hanoi, Vietnam \\ ${ }^{2}$ Institute of Mechanics, VAST, Hanoi, Vietnam \\ *E-mail: ntkhiem@imech.vast.vn
}

Received: 12 September 2018 / Published online: 29 March 2019

\begin{abstract}
The natural frequencies or related resonant frequencies have been widely used for crack detection in structures by the vibration-based technique. However, antiresonant frequencies, the zeros of frequency response function, are less involved to use for the problem because they have not been thoroughly studied. The present paper addresses analysis of antiresonant frequencies of multiple cracked bar in comparison with the resonant ones. First, exact characteristic equations for the resonant and antiresonant frequencies of bar with arbitrary number of cracks are conducted in a new form that is explicitly expressed in term of crack severities. Then, the conducted equations are employed for analysis of variation of resonant and antiresonant frequencies versus crack position and depth. Numerical results show that antiresonant frequencies are indeed useful indicators for crack detection in bar mutually with the resonant ones.

Keywords: multi-cracked bar; longitudinal vibration; frequency equation; antiresonant frequency.
\end{abstract}

\section{INTRODUCTION}

Natural frequencies of a structure are an important dynamical characteristic that is usually computed by solving the so-called characteristic or frequency equation of the structure. Establishing the frequency equation for a structure gets to be crucial for both the analysis and identification of the structure. Adams et al. [1] are the first authors who established exact frequency equation for bar with single crack adopted by the spring model. Narkis [2] and Morassi [3] first obtained closed form solution in locating a crack using frequency equation of longitudinal vibration. More comprehensive study on both the forward and inverse problems in free vibration of multiple cracked bar was accomplished in References [4-10]. However, the study showed that unique solution of the crack detection cannot be found by using only natural frequencies. Some efforts have been made to solve the problem by encompassing other vibration characteristics such mode shapes [11-13] or frequency response function [14], but it was successful when antiresonant frequencies have been employed [15-17]. Nevertheless, using additionally the

(c) 2019 Vietnam Academy of Science and Technology 
antiresonant frequencies for crack detection in bar enables to obtain unique solution of the crack detection problem only for free end bar. This may be caused from that the antiresonant frequencies of cracked bar with different boundary conditions have not been exhaustively investigated.

The present paper is devoted to study systematically variation of antiresonant frequencies of bar versus crack parameters mutually with resonant frequencies. First, there is derived a new form of characteristic equations for both resonant and antiresonant frequencies of multiple cracked bars. Then, the established equations are used for investigating change in the frequencies caused by presence of cracks. Numerical results have been examined to illustration of the proposed herein theory.

\section{GENERAL FREQUENCY EQUATION FOR MULTIPLE CRACKED BAR}

Let's consider longitudinal vibration in a bar that is described by the equation [14]

$$
\Phi^{\prime \prime}(x)+\lambda^{2} \Phi(x)=0, x \in(0,1), \lambda=\omega L \sqrt{\rho / E},
$$

under general boundary conditions

$$
\alpha_{0} \Phi(0)+\beta_{0} \Phi^{\prime}(0)=0, \quad \alpha_{1} \Phi(1)+\beta_{1} \Phi^{\prime}(1)=0,
$$

with the material, geometry and boundary constants $E, \rho, L, \alpha_{0}, \beta_{0}, \alpha_{1}, \beta_{1}$. Suppose that the bar is damaged to crack at arbitrary number $n$ of positions $e_{j}: 0 \leq e_{1}<\ldots<e_{n} \leq 1$. For cracks modeled by transitional spring of stiffness $K_{j}$, conditions at the crack positions are [18]

$$
\begin{gathered}
\Phi^{\prime}\left(e_{j}+0\right)=\Phi^{\prime}\left(e_{j}-0\right), \Phi\left(e_{j}+0\right)=\Phi\left(e_{j}-0\right)+\gamma_{j} \Phi^{\prime}\left(e_{j}\right), \\
\gamma_{j}=E A / L K_{j}=2\left(1-v^{2}\right)(h / L) \theta\left(a_{j} / h\right), j=1, \ldots, n, \\
\theta(z)=0.9852 z^{2}+0.2381 z^{3}-1.0368 z^{4}+1.2055 z^{5}+0.5803 z^{6}-1.0368 z^{7}+0.7314 z^{8} .
\end{gathered}
$$

It can be shown that any solution of equation (1) satisfying the first boundary condition in (2) at $x=0$ and conditions (3) inside the bar is expressed in the form [14]

$$
\Phi(x)=C L(x, \lambda)
$$

where $C$ is a constant and function

$$
\begin{gathered}
L(\lambda x)=L_{0}(\lambda x)+\sum_{k=1}^{n} \mu_{k} K\left(x-e_{k}\right), \\
K(x)=\left\{\begin{array}{ll}
0 & \text { for } x<0 \\
\cos \lambda x & \text { for } x \geq 0
\end{array}, K^{\prime}(x)=\left\{\begin{array}{ll}
0 & \text { for } x<0 \\
-\lambda \sin \lambda x & \text { for } x \geq 0
\end{array},\right.\right. \\
L_{0}(\lambda x)=\left(\alpha_{0} \sin \lambda x-\lambda \beta_{0} \cos \lambda x\right), \\
\mu_{j}=\gamma_{j}\left[L_{0}^{\prime}\left(\lambda e_{j}\right)-\lambda \sum_{k=1}^{j-1} \mu_{k} \sin \lambda\left(e_{j}-e_{k}\right)\right], j=1, \ldots, n .
\end{gathered}
$$

Substituting expression (5) into the second boundary condition in (2) at $x=1$ yields

$$
C\left[\alpha_{1} L(1, \lambda)+\beta_{1} L^{\prime}(1, \lambda)\right]=0
$$


that would have nontrivial solution with respect to constants $C$ under the condition

$$
D(\lambda) \equiv d_{0}(\lambda)+\sum_{j=1}^{n} H\left(1-e_{j}\right) \mu_{j}=0,
$$

where $d_{0}(\lambda)=\alpha_{1} L_{0}(\lambda)+\beta_{1} L_{0}^{\prime}(\lambda) ; H(x)=\alpha_{1} \cos \lambda x-\lambda \beta_{1} \sin \lambda x$. The Eq. (8) is general form of frequency equation for multiple cracked bar that in combination with Eq. (7) enables to compute eigenvalues $\lambda_{1}, \lambda_{2}, \lambda_{3}, \ldots$ dependently on crack parameters. The obtained equation is implicit regarding crack magnitudes $\gamma_{1}, \ldots, \gamma_{n}$, so that solving that equation with respect to the eigenvalues or natural frequencies needs to compute the socalled damage parameters $\mu_{1}, \ldots, \mu_{n}$ defined by Eqs. (7). It would be much simplified in solution of both the forward and inverse problems for cracked bar if an explicit expression of the characteristic equation regarding the crack magnitudes $\gamma_{1}, \ldots, \gamma_{n}$ is available. Indeed, the recurrent relationships (7) can be rewritten as

$$
\begin{aligned}
\mu_{1} & =\gamma_{1} L^{\prime}{ }_{0}\left(\lambda e_{1}\right), \\
\mu_{2}=\gamma_{2} L^{\prime}{ }_{0}\left(\lambda e_{2}\right) & -\lambda \gamma_{1} \gamma_{2} L_{0}^{\prime}\left(\lambda e_{1}\right) \sin \lambda\left(e_{2}-e_{1}\right), \\
\mu_{3}=\gamma_{3} L^{\prime}{ }_{0}\left(\lambda e_{3}\right) & -\lambda \gamma_{1} \gamma_{3} L_{0}^{\prime}\left(\lambda e_{1}\right) \sin \lambda\left(e_{3}-e_{1}\right)-\lambda \gamma_{2} \gamma_{3} L^{\prime}{ }_{0}\left(\lambda e_{2}\right) \sin \lambda\left(e_{3}-e_{2}\right) \\
& +\lambda^{2} \gamma_{1} \gamma_{2} \gamma_{3} L_{0}^{\prime}{ }_{0}\left(\lambda e_{1}\right) \sin \lambda\left(e_{2}-e_{1}\right) \sin \lambda\left(e_{3}-e_{2}\right),
\end{aligned}
$$

Substituting latter expressions into (8) one obtains

$$
\begin{aligned}
D(\lambda) \equiv & d_{0}(\lambda)+\sum_{j=1}^{n} \gamma_{j} d_{1}\left(\lambda, e_{j}\right)-\lambda \sum_{j=2}^{n} \sum_{k=1}^{j-1} d_{2}\left(\lambda, e_{j}, e_{k}\right) \gamma_{j} \gamma_{k} \\
& +\lambda^{2} \sum_{j=3}^{n} \sum_{k=2}^{j-1} \sum_{r=1}^{k-1} d_{3}\left(\lambda, e_{j}, e_{k}, e_{r}\right) \gamma_{j} \gamma_{k} \gamma_{r}+\ldots+(-\lambda)^{n-1} d_{n}\left(\lambda, e_{n}, \ldots, e_{1}\right) \gamma_{1} \gamma_{2} \ldots \gamma_{n} \\
= & d_{0}(\lambda)+\sum_{k=1}^{n} \sum_{1 \leq i_{1}<i_{2}<\ldots<i_{k} \leq n}(-\lambda)^{k-1} d_{k}\left(\lambda, e_{i_{k}}, e_{i_{k-1}}, \ldots, e_{i_{1}}\right) \gamma_{i_{1}} \gamma_{i_{2}} \ldots \gamma_{i_{k}}=0
\end{aligned}
$$

where

$$
\begin{aligned}
& d_{0}(\lambda)=\alpha_{1} L_{0}(\lambda)+\beta_{1} L_{0}^{\prime}(\lambda), \\
& d_{1}\left(\lambda, e_{j}\right)=H\left(1-e_{j}\right) L_{0}^{\prime}\left(\lambda e_{j}\right), \\
& d_{2}\left(\lambda, e_{j}, e_{k}\right)=H\left(1-e_{j}\right) \sin \lambda\left(e_{j}-e_{k}\right) L_{0}^{\prime}\left(\lambda e_{k}\right), \\
& d_{3}\left(\lambda, e_{j}, e_{k}, e_{r}\right)=H\left(1-e_{j}\right) \sin \lambda\left(e_{j}-e_{k}\right) \sin \lambda\left(e_{k}-e_{r}\right) L_{0}^{\prime}\left(\lambda e_{r}\right), \\
& d_{n}\left(\lambda, e_{n}, \ldots, e_{1}\right)=H\left(1-e_{n}\right) \sin \lambda\left(e_{n}-e_{n-1}\right) \sin \lambda\left(e_{n-1}-e_{n-2}\right) \sin \lambda\left(e_{2}-e_{1}\right) L_{0}^{\prime}\left(\lambda e_{1}\right) .
\end{aligned}
$$

The obtained equation (9) is desired explicit form of the characteristic equation that provides an efficient tool for solving not only the forward but also the inverse problem of multiple cracked bar. Note, another form of the characteristic equation for multiple 
cracked rod was exactly obtained by Shifrin in [7], but that equation is implicit with respect to the crack magnitudes, likely, the equation given by Khiem et al. in [14].

In the case of intact bar, Eq. (9) becomes

$$
d_{0}(\lambda) \equiv\left(\alpha_{0} \alpha_{1}+\lambda^{2} \beta_{0} \beta_{1}\right) \sin \lambda-\lambda\left(\alpha_{1} \beta_{0}-\alpha_{0} \beta_{1}\right) \cos \lambda=0
$$

Next, for bar with single, double and triple cracks exact frequency equations get respectively the forms

$$
\begin{aligned}
D_{1}(\lambda) \equiv & d_{0}(\lambda)+\gamma_{1} d_{1}\left(\lambda, e_{1}\right)=0, \\
D_{2}(\lambda) \equiv & d_{0}(\lambda)+\gamma_{1} d_{1}\left(\lambda, e_{1}\right)+\gamma_{2} d_{1}\left(\lambda, e_{2}\right)-\lambda \gamma_{1} \gamma_{2} d_{2}\left(\lambda, e_{2}, e_{1}\right)=0, \\
D_{3}(\lambda) \equiv & d_{0}(\lambda)+\gamma_{1} d_{1}\left(\lambda, e_{1}\right)+\gamma_{2} d_{1}\left(\lambda, e_{2}\right)+\gamma_{3} d_{1}\left(\lambda, e_{3}\right)-\lambda \gamma_{1} \gamma_{2} d_{2}\left(\lambda, e_{2}, e_{1}\right), \\
& -\lambda \gamma_{1} \gamma_{3} d_{2}\left(\lambda, e_{3}, e_{1}\right)-\lambda \gamma_{2} \gamma_{3} d_{2}\left(\lambda, e_{3}, e_{2}\right)+\lambda^{2} \gamma_{1} \gamma_{2} \gamma_{3} d_{3}\left(\lambda, e_{3}, e_{2}, e_{1}\right)=0 .
\end{aligned}
$$

Moreover, if the cracks are small so that asymptotic approximations of first, second and third order respectively for the frequency equation are

$$
\begin{aligned}
& d_{0}(\lambda)+\sum_{j=1}^{n} \gamma_{j} d_{1}\left(e_{j}\right)=0 \\
& d_{0}(\lambda)+\sum_{j=1}^{n} \gamma_{j} d_{1}\left(e_{j}\right)-\lambda \sum_{j=2}^{n} \sum_{k=1}^{j-1} d_{2}\left(\lambda, e_{j}, e_{k}\right) \gamma_{j} \gamma_{k}=0 \\
& d_{0}(\lambda)+\sum_{j=1}^{n} \gamma_{j} d_{1}\left(e_{j}\right)-\lambda \sum_{j=2}^{n} \sum_{k=1}^{j-1} d_{2}\left(\lambda, e_{j}, e_{k}\right) \gamma_{j} \gamma_{k}+\lambda^{2} \sum_{j=3}^{n} \sum_{k=2}^{j-1} \sum_{r=1}^{k-1} d_{3}\left(\lambda, e_{j}, e_{k}, e_{r}\right) \gamma_{j} \gamma_{k} \gamma_{r}=0
\end{aligned}
$$

Finally, it has to note that general boundary conditions (2) include all the conventional end conditions and the elastic ones in dependence on the specific combinations of parameters $\left(\alpha_{0}, \beta_{0}, \alpha_{1}, \beta_{1}\right)$. Namely, for the case of free-free ends, (a) $\Phi^{\prime}(0)=\Phi^{\prime}(1)=0$; fixed ends, (b) $\Phi(0)=\Phi(1)=0$ and fixed-free ends, (c) $\Phi(0)=\Phi^{\prime}(1)=0$, the parameters get respectively

(a) $\alpha_{0}=\alpha_{1}=0, \beta_{0}=\beta_{1}=1$, (b) $\alpha_{0}=\alpha_{1}=1, \beta_{0}=\beta_{1}=0$, (c) $\beta_{0}=\alpha_{1}=0, \alpha_{0}=\beta_{1}=1$.

So that, for the listed above boundary conditions one has

(a) Free-Free ends: $L_{0}(x)=-\lambda \cos \lambda x, L_{0}^{\prime}(x)=\lambda^{2} \sin \lambda x, H_{1}(x)=-\lambda \sin \lambda x$, and therefore

$$
\begin{aligned}
d_{0}(\lambda) & =\lambda^{2} \sin \lambda, \\
d_{1}(\lambda, e) & =-\lambda^{3} \sin \lambda e \sin \lambda(1-e), \\
d_{2}\left(\lambda, e_{1}, e_{2}\right) & =-\lambda^{3} \sin \lambda e_{1} \sin \lambda\left(e_{2}-e_{1}\right) \sin \lambda\left(1-e_{2}\right),
\end{aligned}
$$


(b) Fixed-Fixed ends: $L_{0}(x)=\sin \lambda x, L_{0}^{\prime}(x)=\lambda \cos \lambda x, H_{1}(x)=\cos \lambda x$ and

$$
\begin{aligned}
d_{0}(\lambda) & =\sin \lambda, \\
d_{1}(\lambda, e) & =\cos \lambda(1-e) \sin \lambda e, \\
d_{2}\left(\lambda, e_{1}, e_{2}\right) & =\lambda \cos \lambda\left(1-e_{2}\right) \sin \lambda\left(e_{2}-e_{1}\right) \cos \lambda e_{1} .
\end{aligned}
$$

(c) Fixed-Free ends: $L_{0}(x)=\sin \lambda x, L_{0}^{\prime}(x)=\lambda \cos \lambda x, H_{1}(x)=-\lambda \sin \lambda x$ and

$$
\begin{aligned}
d_{0}(\lambda) & =\lambda \cos \lambda x, \\
d_{1}(\lambda, e) & =-\lambda^{2} \sin \lambda(1-e) \cos \lambda e, \\
d_{2}\left(\lambda, e_{1}, e_{2}\right) & =-\lambda^{2} \sin \lambda\left(1-e_{2}\right) \sin \lambda\left(e_{2}-e_{1}\right) \cos \lambda e_{1} .
\end{aligned}
$$

If both the ends of bar are supported by translational springs of stiffness $S_{0}, S_{1}$, the parameters $\left(\alpha_{0}, \beta_{0}, \alpha_{1}, \beta_{1}\right)$ are defined as $\alpha_{0}=\alpha_{1}=1, \beta_{0}=-E A / S_{0}, \beta_{1}=E A / S_{1}$, so that

$L_{0}(x)=\sin \lambda x+\lambda \beta_{0} \cos \lambda x, L_{0}^{\prime}(x)=\lambda \cos \lambda x-\lambda^{2} \beta_{0} \sin \lambda x, H_{1}(x)=\cos \lambda x-\lambda \beta_{1} \sin \lambda x$, and

$$
\begin{aligned}
d_{0}(\lambda) & =\lambda\left(\beta_{0}+\beta_{1}\right) \cos \lambda+\left(1-\lambda^{2} \beta_{0} \beta_{1}\right) \sin \lambda \\
d_{1}(\lambda, e) & =\lambda\left[\cos \lambda(1-e)-\lambda \beta_{1} \sin \lambda(1-e)\right]\left(\cos \lambda e-\lambda \beta_{0} \sin \lambda e\right), \\
d_{2}\left(\lambda, e_{1}, e_{2}\right) & =\lambda\left[\cos \lambda\left(1-e_{2}\right)-\lambda \beta_{1} \sin \lambda\left(1-e_{2}\right)\right]\left(\cos \lambda e_{1}-\lambda \beta_{0} \sin \lambda e_{1}\right) \sin \lambda\left(e_{2}-e_{1}\right) .
\end{aligned}
$$

Moreover, in the case of small cracks first order asymptotic approximations of the frequency equation for the conventional (Free-Free; Fixed-Fixed; Fixed-Free) boundary conditions are

$$
\begin{aligned}
& \sin \lambda-\lambda \sum_{j=1}^{n} \gamma_{j} \sin \lambda e_{j} \sin \lambda\left(1-e_{j}\right)=0, \\
& \sin \lambda+\lambda \sum_{j=1}^{n} \gamma_{j} \cos \lambda e_{j} \cos \lambda\left(1-e_{j}\right)=0, \\
& \cos \lambda-\lambda \sum_{j=1}^{n} \gamma_{j} \cos \lambda e_{j} \sin \lambda\left(1-e_{j}\right)=0 .
\end{aligned}
$$

Also, assuming $\lambda=\lambda_{0}+\Delta \lambda$ with $\lambda_{0}$ being the frequency parameter of intact bar and small $\Delta \lambda$, the latter equations yield all those obtained in earlier studies, for example, [8].

\section{ANTIRESONANT FREQUENCY EQUATION FOR MULTIPLE CRACKED BARS}

As well known in the vibration theory, resonant frequencies of a mechanical system are poles of the system's frequency Response Function (FRF) while antiresonant ones are zeros of the FRF. In case of systems without damping, the resonant frequencies are identical to natural frequencies determined as roots of the frequency equations. As zeros of FRF, antiresonant frequencies of a multiple cracked bar are seeking as follows. General 
expression of FRF for multiple cracked bars has been obtained by Khiem et al. $[12,13]$ in the form

$F R F\left(\omega, x_{0}, x\right)=\frac{L}{\lambda E F}\left\{\sin \lambda\left(x-x_{0}\right)-\frac{g\left(1-x_{0}\right)\left[\alpha_{0} \sin \lambda x-\beta_{0} \lambda \cos \lambda x+\sum_{j=1}^{n} \mu_{j} S\left(x-e_{j}\right)\right]}{d_{0}(\lambda)+\sum_{j=1}^{n} \mu_{j}\left[\alpha_{1} \cos \lambda\left(1-e_{j}\right)-\beta_{1} \lambda \sin \lambda\left(1-e_{j}\right)\right]}\right\}$,

where $g(x)=\alpha_{1} \sin \lambda x+\beta_{1} \lambda \cos \lambda x$ and function $S(x)=\{0$ if $x<0 ; \cos \lambda x$ if $x \geq 0\}$. Letting $x=x_{0}=1$, that implies the FRF determined with both the input and output applied at the right end of bar, Eq. (26) is simplified to

$$
F R F(\omega, 1,1)=\frac{-\beta_{1} L}{E F} \frac{\left[\alpha_{0} \sin \lambda-\beta_{0} \lambda \cos \lambda+\sum_{j=1}^{n} \mu_{j} \cos \lambda\left(1-e_{j}\right)\right]}{d_{0}(\lambda)+\sum_{j=1}^{n} \mu_{j}\left[\alpha_{1} \cos \lambda\left(1-e_{j}\right)-\beta_{1} \lambda \sin \lambda\left(1-e_{j}\right)\right]} .
$$

Therefore, antiresonant frequencies can be sought by solving the equation

$$
\alpha_{0} \sin \bar{\lambda}-\beta_{0} \bar{\lambda} \cos \bar{\lambda}+\sum_{j=1}^{n} \mu_{j} \cos \bar{\lambda}\left(1-e_{j}\right)=0,
$$

with respect to $\bar{\lambda}$ or

$$
\bar{d}_{0}(\bar{\lambda})+\sum_{j=1}^{n} \bar{H}\left(1-e_{j}\right) \mu_{j}=0,
$$

where $\bar{d}_{0}(\bar{\lambda})=\alpha_{0} \sin \bar{\lambda}-\beta_{0} \bar{\lambda} \cos \bar{\lambda} ; \bar{H}(x)=\cos \bar{\lambda} x$. Eq. (28) has the same form as Eq. (8) where the functions $d_{0}(\lambda), H(x)$ are replaced by $\bar{d}_{0}(\lambda), \bar{H}(x)$ and in both the equations the parameters $\mu_{j}$ are expressed by the same equations (7). Thus, equation for antiresonant frequencies (called herein antiresonant frequency equation) can be derived as

$$
\bar{d}_{0}(\bar{\lambda})+\sum_{k=1}^{n} \sum_{1 \leq i_{1}<i_{2}<\ldots<i_{k} \leq n}(-\bar{\lambda})^{k-1} \bar{d}_{k}\left(\bar{\lambda}, e_{i_{1}}, e_{i_{2}}, \ldots, e_{i_{k}}\right) \gamma_{i_{1}} \gamma_{i_{2}} \ldots \gamma_{i_{k}}=0
$$

where

$$
\begin{aligned}
& \bar{d}_{1}\left(\lambda, e_{j}\right)=\bar{H}\left(1-e_{j}\right) L_{0}^{\prime}\left(\bar{\lambda} e_{j}\right), \\
& \bar{d}_{2}\left(\bar{\lambda}, e_{j}, e_{k}\right)=\bar{H}\left(1-e_{j}\right) \sin \bar{\lambda}\left(e_{j}-e_{k}\right) L^{\prime}{ }_{0}\left(\bar{\lambda} e_{k}\right), \\
& \bar{d}_{3}\left(\lambda, e_{j}, e_{k}, e_{r}\right)=\bar{H}\left(1-e_{j}\right) \sin \bar{\lambda}\left(e_{j}-e_{k}\right) \sin \bar{\lambda}\left(e_{k}-e_{r}\right) L_{0}^{\prime}\left(\bar{\lambda} e_{r}\right), \\
& \bar{d}_{n}\left(\bar{\lambda}, e_{n}, \ldots, e_{1}\right)=\bar{H}\left(1-e_{n}\right) \sin \bar{\lambda}\left(e_{n}-e_{n-1}\right) \sin \bar{\lambda}\left(e_{n-1}-e_{n-2}\right) \sin \bar{\lambda}\left(e_{2}-e_{1}\right) L^{\prime}\left(\bar{\lambda} e_{1}\right) .
\end{aligned}
$$


Similarly, the first, second and third order asymptotic approximations of the antiresonant frequency equation can be obtained respectively as

$$
\begin{aligned}
& \bar{d}_{0}(\bar{\lambda})+\sum_{j=1}^{n} \gamma_{j} \bar{d}_{1}\left(\bar{\lambda}, e_{j}\right)=0 \\
& \bar{d}_{0}(\bar{\lambda})+\sum_{j=1}^{n} \gamma_{j} \bar{d}_{1}\left(\bar{\lambda}, e_{j}\right)-\bar{\lambda} \sum_{j=2}^{n} \sum_{k=1}^{j-1} \bar{d}_{2}\left(\bar{\lambda}, e_{j}, e_{k}\right) \gamma_{j} \gamma_{k}=0 \\
& \bar{d}_{0}(\bar{\lambda})+\sum_{j=1}^{n} \gamma_{j} \bar{d}_{1}\left(\bar{\lambda}, e_{j}\right)-\bar{\lambda} \sum_{j=2}^{n} \sum_{k=1}^{j-1} \bar{d}_{2}\left(\bar{\lambda}, e_{j}, e_{k}\right) \gamma_{j} \gamma_{k}+\bar{\lambda}^{2} \sum_{j=3}^{n} \sum_{k=2}^{j-1} \sum_{r=1}^{k-1} \bar{d}_{3}\left(\bar{\lambda}, e_{j}, e_{k}, e_{r}\right) \gamma_{j} \gamma_{k} \gamma_{r}=0 .
\end{aligned}
$$

Since the FRF (26) is meaningless at the fixed ends of bar, the antiresonant frequency equations (29) and (31)-(33) are applied only for free end bar and cantilever bar. In the latter cases of boundary conditions, the first order approximate antiresonant frequency equation are

$$
\begin{aligned}
& \cos \bar{\lambda}-\bar{\lambda} \sum_{j=1}^{n} \gamma_{j} \cos \bar{\lambda}\left(1-e_{j}\right) \sin \bar{\lambda} e_{j}=0, \\
& \sin \bar{\lambda}+\bar{\lambda} \sum_{j=1}^{n} \gamma_{j} \cos \bar{\lambda}\left(1-e_{j}\right) \cos \bar{\lambda} e_{j}=0 .
\end{aligned}
$$

Eq. (35) shows that antiresonant frequencies of fixed-free bar are resonant frequencies of fixed end bar (see Eq. (24), so they are the same for symmetric cracks. Nevertheless, antiresonant frequencies of free-free end bar, likely resonant frequencies of cantilever bar, have not the symmetric effect. The latter fact has been employed by Rubio et al. [17] to obtain unique solution in localization of single and double crack in free-free end rod from given resonant and antiresonant frequencies. However, as shown below, the result cannot be extended for other cases of boundary conditions, even if other pair of resonant and antiresonant frequencies are used.

To validate the proposed theoretical development, antiresonant frequencies of the free end bar that was experimentally examined by the authors of Ref. [15] are computed and compared to the measured ones (see Tab. 1).

Obviously, calculated and measured antiresonant frequencies are excellently agreed (descrepancy between them is less than $1 \%$. However, the descrepancy increases with severity of damage, esspecially, for higher frequencies. Note, deviation between calculated and measured first antiresonant frequency is of the same order $7 \%$ for both the cases of damage severity $D_{1}$ and $D_{2}$. This is perhaps caused by inaccuracy of the crack model used for representing the saw cut in the experimentation.

\section{CRACK-INDUCED CHANGE IN RESONANT AND ANTIRESONANT FREQUENCIES (NUMERICAL RESULTS)}

The problem of single crack detection in free end bar has been thoroughly studied by Morassi and his coworkers. However, it is necessary to note that the unique solution 
Table 1. Antiresonant frequencies of intact and cracked bar compared to the measured ones

\begin{tabular}{ccccccc}
\hline \multirow{2}{*}{$\begin{array}{c}\text { Mode } \\
\text { No }\end{array}$} & \multicolumn{2}{c}{ Intact bar } & \multicolumn{2}{c}{ Damage senario $\mathrm{D}_{1}$} & \multicolumn{2}{c}{ Damage senario $\mathrm{D}_{2}$} \\
\cline { 2 - 7 } & \multicolumn{1}{c}{ Exp. } & $\begin{array}{c}\text { Present } \\
\text { (deviation, \%) }\end{array}$ & \multicolumn{1}{c}{$\begin{array}{c}\text { Exp. } \\
\text { [15] }\end{array}$} & $\begin{array}{c}\text { Present } \\
\text { (deviation, \%) }\end{array}$ & \multicolumn{1}{c}{$\begin{array}{c}\text { Exp. } \\
{[15]}\end{array}$} & $\begin{array}{c}\text { Present } \\
\text { (deviation, \%) }\end{array}$ \\
\hline 1 & 468.6 & $470.6(0.42)$ & 439.5 & $470.3(7.0)$ & 432.9 & $465.3(7.48)$ \\
2 & 1411.7 & $1411.7(0)$ & 1409.3 & $1406.4(0.2)$ & 1365.6 & $1301.7(4.67)$ \\
3 & 2328.4 & $2352.8(1.05)$ & 2337.0 & $2339.6(0.1)$ & 2324.4 & $2132.9(8.23)$ \\
4 & 3265.8 & $3294.0(0.86)$ & - & $3282.1(-)$ & 3102.5 & $3134.1(1.01)$ \\
5 & 4216.6 & $4235.1(0.43)$ & - & $4232.9(-)$ & 3722.1 & $4200.8(12.86)$ \\
6 & 5145.1 & $5176.3(0.67)$ & - & $5173.3(-)$ & 4866.6 & $5098.5(4.76)$ \\
\hline
\end{tabular}

Damage scenarios $\mathrm{D}_{1}, \mathrm{D}_{2}$ correspond to different depth $(6$ and $15 \mathrm{~mm})$ of crack at position $e=0.55 / 2.747$

in locating single crack was attained in [17] because only first resonant and antiresonant frequencies have been used. The unique solution could not be obtained by using a pair of second or higher resonant and antiresonant frequencies. Obviously, the resonant and antiresonant frequencies used for obtaining unique solution have no critical point, crack occurred at which do not change the frequencies. Consequently, it can be expected that existence of the critical points for resonant and antiresonant frequencies destroys the uniqueness of solution in crack detection problem by using the frequencies. Therefore, knowing the critical points, that are called hereby nodes of resonant and antiresonant frequencies, is important in solving the crack detection problem.

The above equations show that nodes of resonant frequencies can be sought from equation $d_{1}\left(\lambda_{0}, x\right)=0$, where $\lambda_{0}$ is solution of frequency equation in case of uncracked bar. Nodes of five lowest resonant frequencies are given in Tab. 2 for the cases of classical boundary conditions.

Table 2. Nodes of resonant frequencies for bar with classical boundary conditions

\begin{tabular}{|c|c|c|c|}
\hline $\begin{array}{c}\text { Mode } \\
\text { No }\end{array}$ & Fixed end bar & Free-free end bar & Fixed-free end bar \\
\hline 1 & $1 / 2$ & not available & not available \\
\hline 2 & $1 / 4 \quad 3 / 4$ & 0.5 & $1 / 3$ \\
\hline 3 & $\begin{array}{lll}1 / 6 & 1 / 2 & 5 / 6\end{array}$ & $1 / 3 \quad 2 / 3$ & $0.2 \quad 0.6$ \\
\hline 4 & $\begin{array}{llll}1 / 8 & 3 / 8 & 5 / 8 & 7 / 8\end{array}$ & $0.25 \quad 0.5 \quad 0.75$ & $1 / 7 \quad 3 / 7 \quad 5 / 7$ \\
\hline 5 & $1 / 10 \quad 3 / 10 \quad 01 / 2 \quad 7 / 10 \quad 9 / 10$ & $\begin{array}{llll}0.2 & 0.4 & 0.6 & 0.8\end{array}$ & $\begin{array}{llll}1 / 9 & 3 / 9 & 5 / 9 & 7 / 9\end{array}$ \\
\hline
\end{tabular}

For finding nodes of antiresonant frequencies of free-free bar and fixed-free bar one has the following equations $\cos \lambda_{0}(1-x) \sin \lambda_{0} x=0$ and $\cos \lambda_{0}(1-x) \cos \lambda_{0} x=0$, respectively. Solutions of the equations for five modes are given in Tab. 3. Evidently, node of resonant frequencies in fixed end bar exactly coincide with nodes of antiresonant frequencies in fixed-free end bar. All the calculated nodes of resonant and antiresonant 
frequencies of the fixed-free (Fig. 1) and free-free (Fig. 2) bars can be observed in Figs. 1-2 where there are shown ratios of the frequencies to those of intact bar. The ratios (resonant on the left and antiresonant - on the right) are plotted versus crack position (from 1 to 1) in different crack depth $(10 \%-50 \%)$.

Table 3. Nodes of antiresonant frequencies for bar with free ends and fixed-free ends

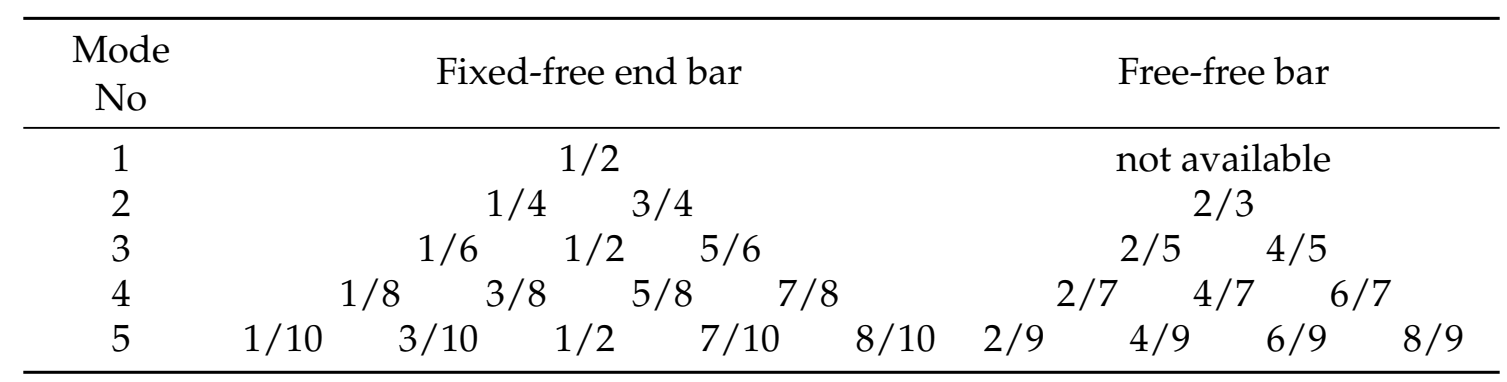
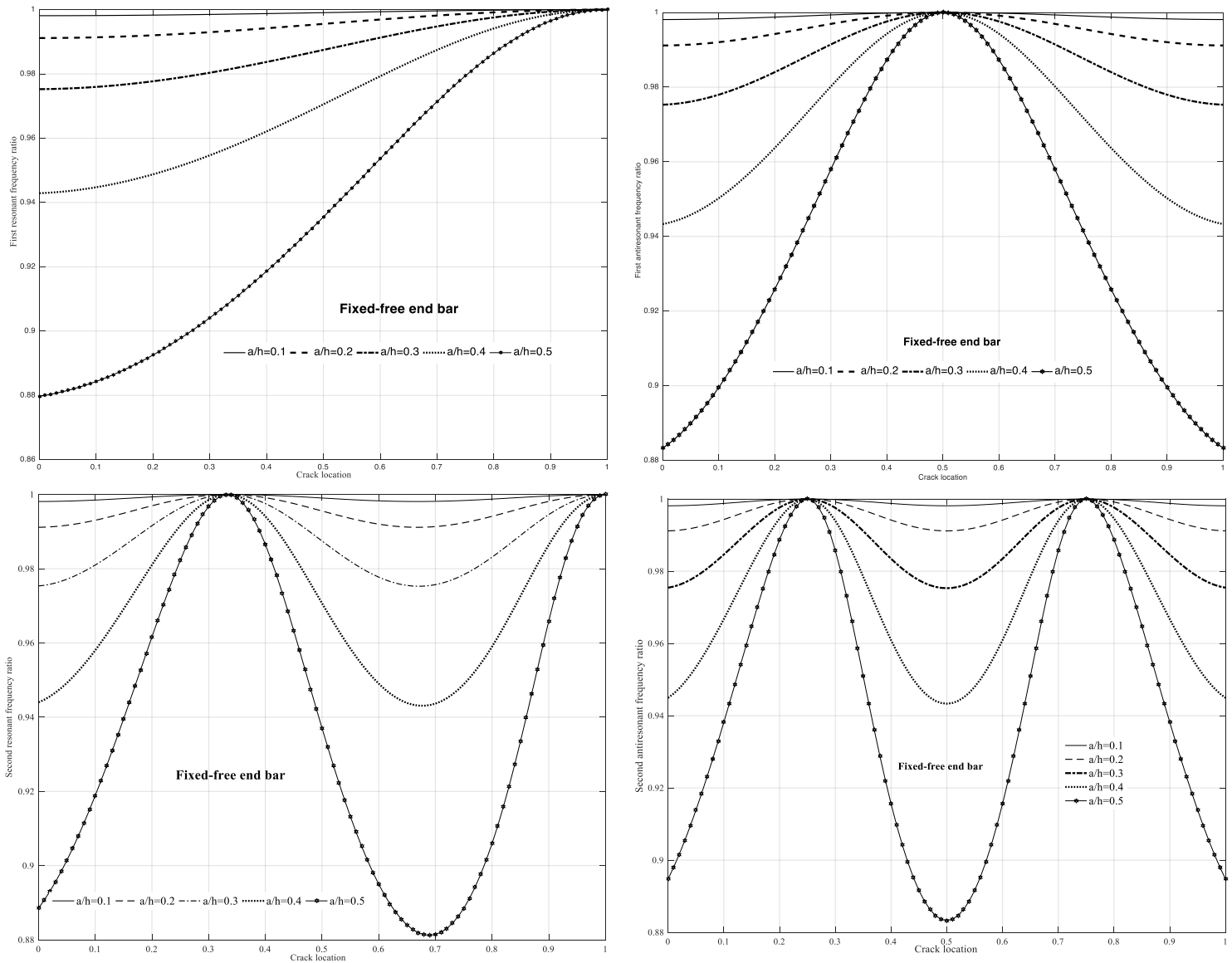

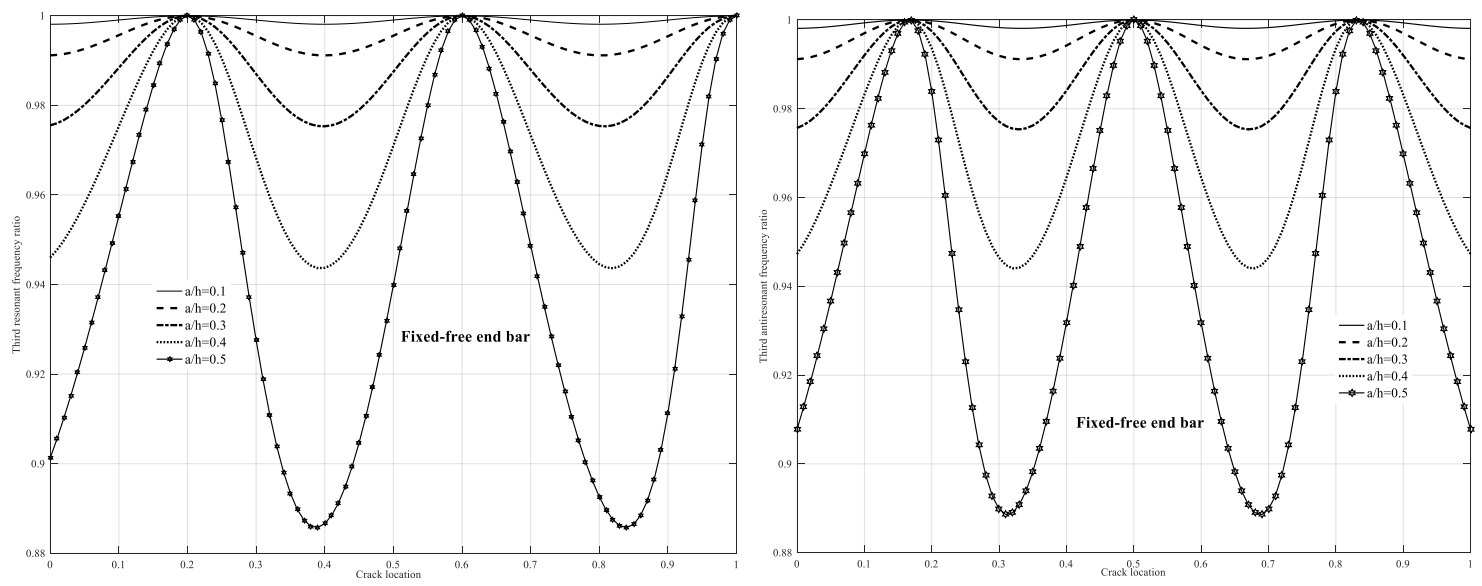

Fig. 1. Variation of three lowest resonant (left) and antiresonant (right) frequencies of fixed-free bar versus crack position with different crack depth $(10 \%-50 \%)$
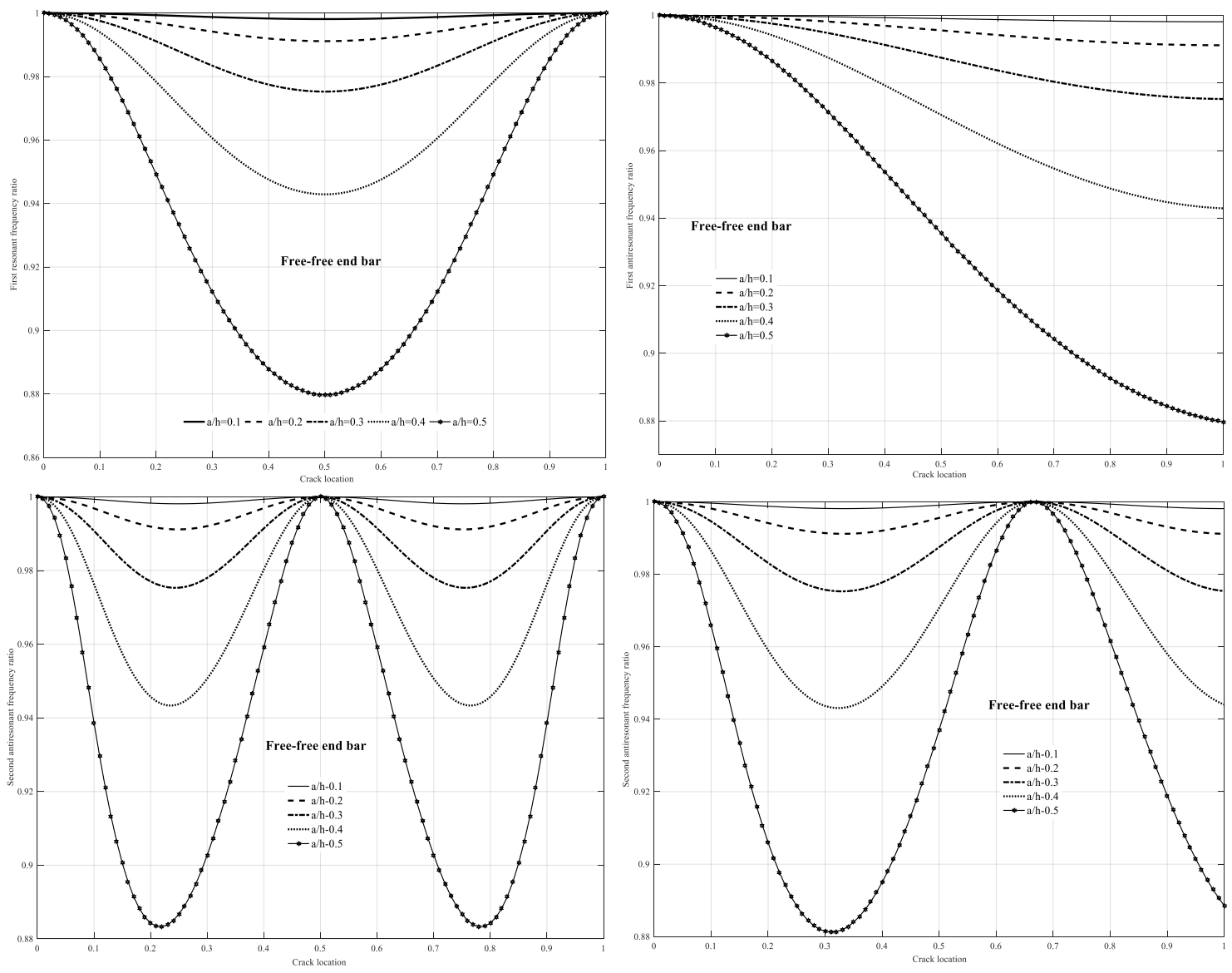

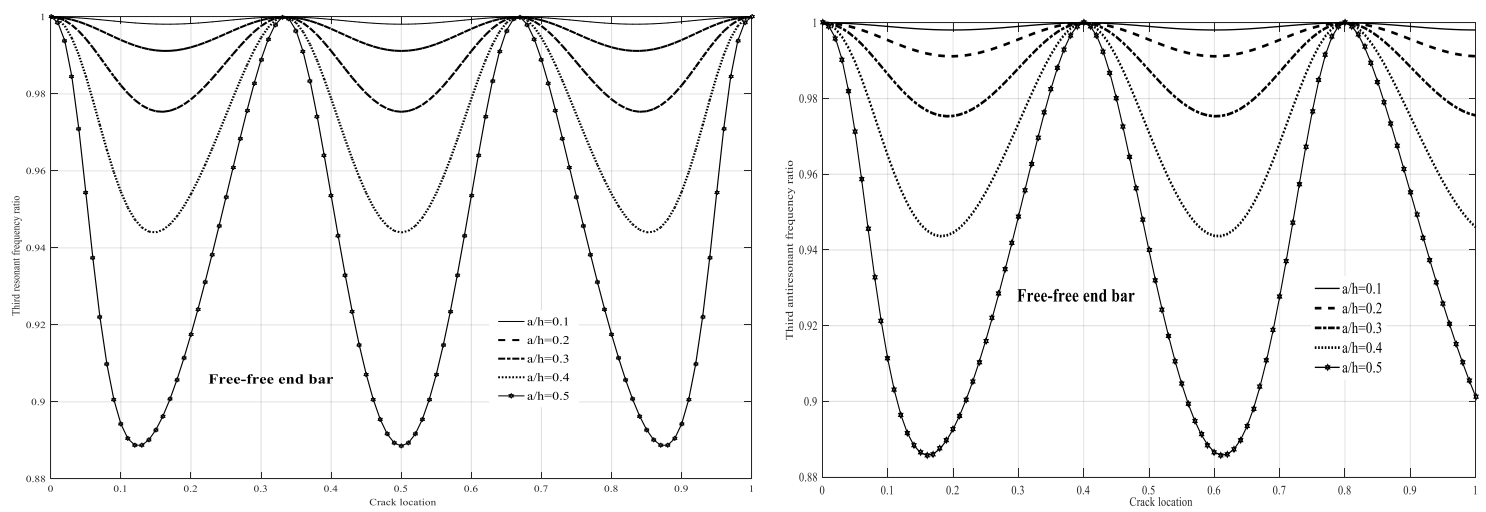

Fig. 2. Variation of three lowest resonant (left) and antiresonant (right) frequencies of free-free bar versus crack position with different crack depth $(10 \%-50 \%)$

Observing graphics given in the Figures demonstrates that crack at free end of bar makes no effect on the resonant frequencies, while it would do significant change in antiresonant frequencies if the frequency response function is defined at this position. Likely to the resonant frequencies, antiresonant frequencies are all monotonically reduced with increasing depth of crack except the nodes (Tab. 3) where they are unaffected by the crack presence.

The ratios of resonant and antiresonant frequencies computed for free-free end bar with two cracks are presented respectively in Figs. 3-4. Obviously, symmetric cracks make the same effect on resonant frequencies of the bar, but this is not true for antiresonant frequencies. Also, the larger number of cracks makes more reduction of antiresonant frequencies.

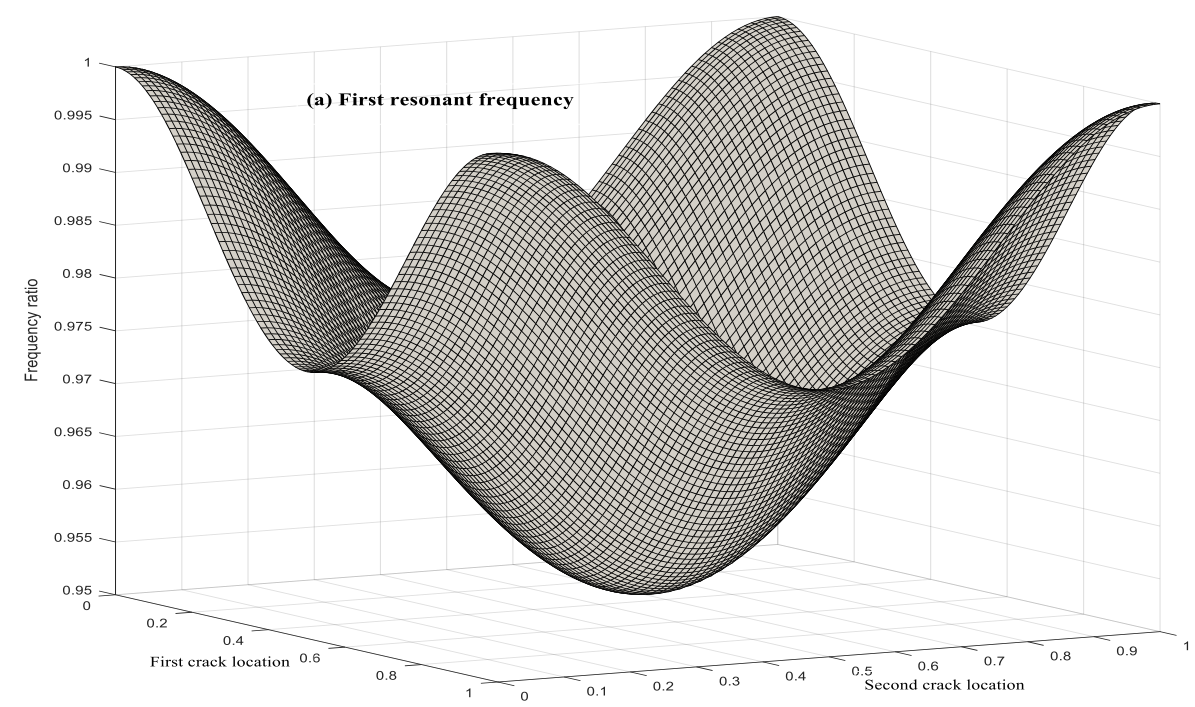




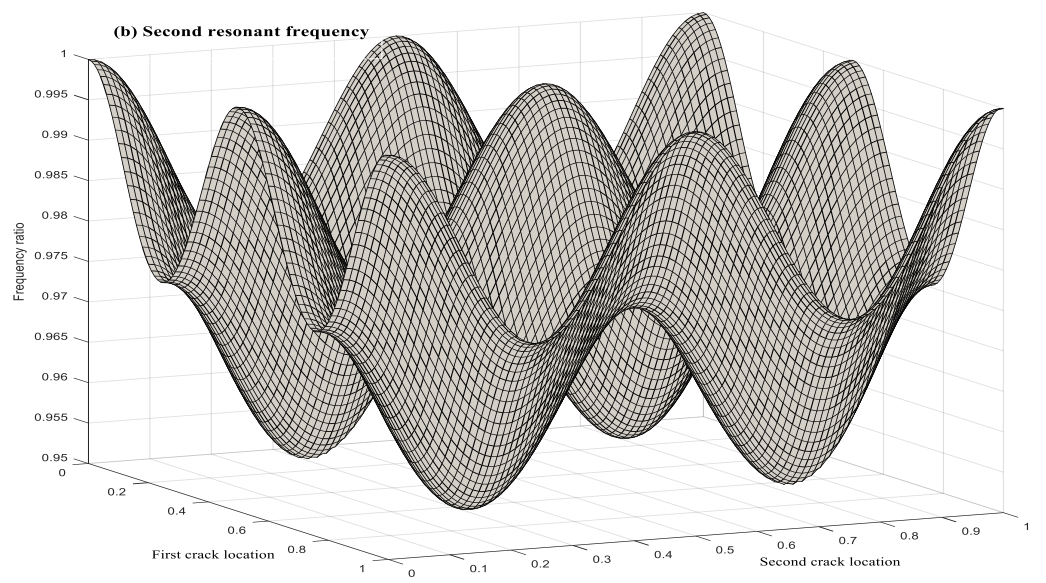

Fig. 3. Variation of first and second resonant frequencies versus position of two cracks with equal depth $30 \%$ for free end bar
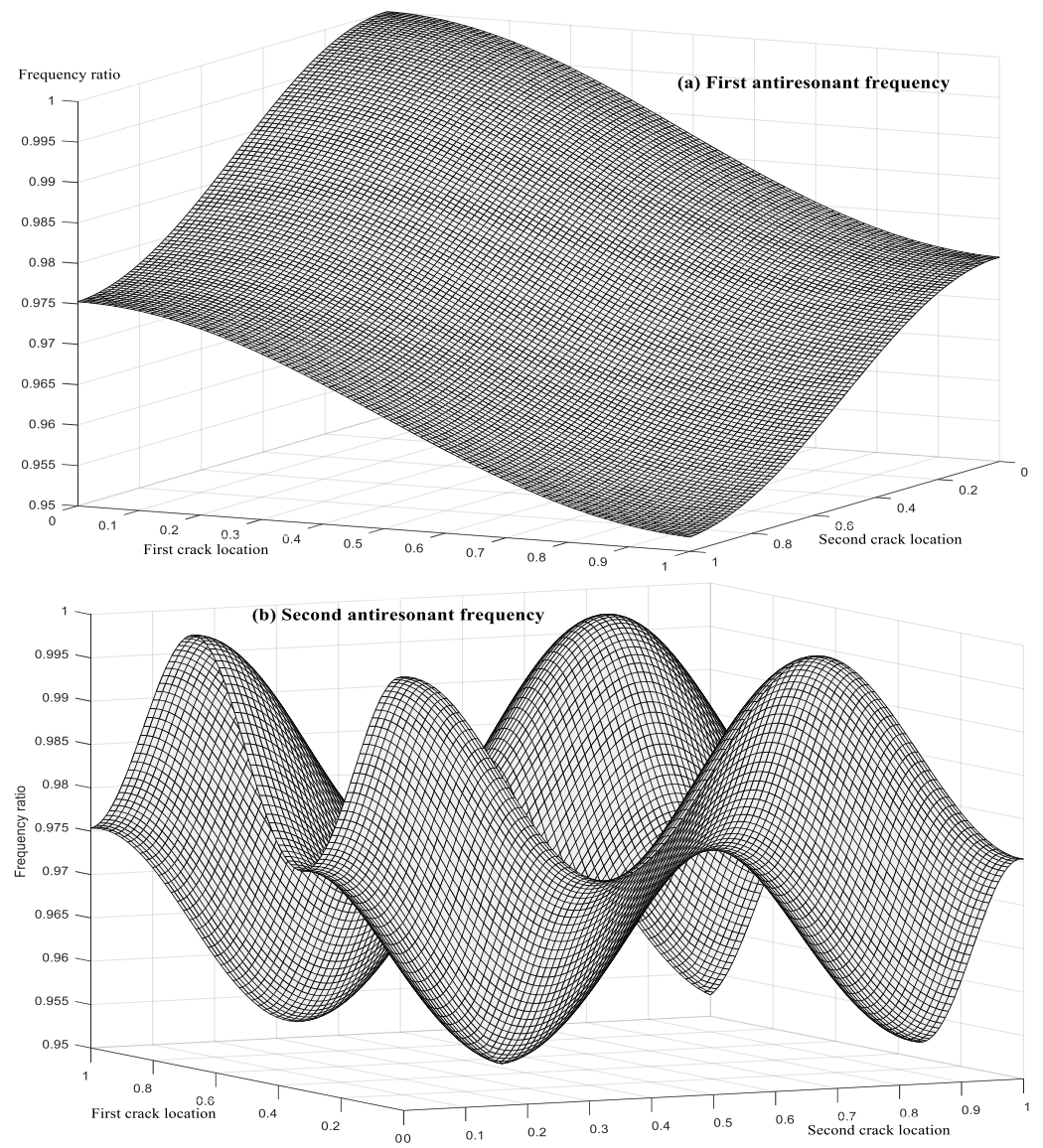

Fig. 4. Variation of first and second antiresonant frequencies versus positions of two cracks with equal depth $30 \%$ for free end bar 


\section{CONCLUSIONS}

In the present work there has been derived a novel form of characteristic equation for resonant and antiresonant frequencies of multiple cracked bar that is explicitly expressed in terms of crack magnitudes. The conducted characteristic equations are general regarding boundary conditions and exact in comparison with the numerous approximate ones known in the literature. These characteristic equations provide a useful tool for developing crack detection procedures in bar.

The antiresonant frequencies of bar with single and double cracks have been examined versus crack position and depth mutually with the resonant ones. The obtained results show that there exist also nodes for antiresonant frequencies but they are different from those of resonant ones. Furthermore, resonant frequencies are defined independently upon where frequency response is measured, while antiresonant frequencies are strongly dependent on the FRF's measurement. Therefore, effect of crack position on an antiresonant frequency may be also different if the antiresonant frequency is extracted from different FRFs. The observed different properties of resonant and antiresonant frequencies may be helpful for detecting cracks in bar by using both of them.

The question that is open in this study is how to determine antiresonant frequencies of cracked bar with fixed-fixed ends. This problem is easily solved for uncracked bar, but it is unsolved for a bar with a crack because the points selected for measurement of FRF may disregard effect of the crack on the FRF. The problem mentioned above is subject for further study of the authors.

\section{ACKNOWLEDGEMENT}

The first author is thankful to University of Transport and Communications for its financial support to complete this study under grant number T2019-CB-013.

The second author is thankful to NAFOSTED of Vietnam for support under grant number 107.02-2015.34 in completing this work.

\section{REFERENCES}

[1] R. D. Adams, P. Cawley, C. J. Pye, and B. J. Stone. A vibration technique for non-destructively assessing the integrity of structures. Journal of Mechanical Engineering Science, 20, (2), (1978), pp. 93-100. https://doi.org/10.1243/jmes_jour_1978_020_016_02.

[2] Y. Narkis. Identification of crack location in vibrating simply supported beams. Journal of Sound and Vibration, 172, (4), (1994), pp. 549-558. https://doi.org/10.1006/jsvi.1994.1195.

[3] A. Morassi. Identification of a crack in a rod based on changes in a pair of natural frequencies. Journal of Sound and Vibration, 242, (4), (2001), pp. 577-596. https://doi.org/10.1006/jsvi.2000.3380.

[4] M. Dilena and A. Morassi. Detecting cracks in a longitudinally vibrating beam with dissipative boundary conditions. Journal of Sound and Vibration, 267, (1), (2003), pp. 87-103. https://doi.org/10.1016/s0022-460x(03)00176-7.

[5] R. Ruotolo and C. Surace. Natural frequencies of a bar with multiple cracks. Journal of Sound and Vibration, 272, (1-2), (2004), pp. 301-316. https://doi.org/10.1016/s0022-460x(03)00761-2. 
[6] L. Rubio, J. Fernández-Sáez, and A. Morassi. Identification of two cracks in a rod by minimal resonant and antiresonant frequency data. Mechanical Systems and Signal Processing, 60, (2015), pp. 1-13. https://doi.org/10.1016/j.ymssp.2015.01.025.

[7] E. I. Shifrin. Inverse spectral problem for a rod with multiple cracks. Mechanical Systems and Signal Processing, 56, (2015), pp. 181-196. https://doi.org/10.1016/j.ymssp.2014.11.004.

[8] E. I. Shifrin. Identification of a finite number of small cracks in a rod using natural frequencies. Mechanical Systems and Signal Processing, 70, (2016), pp. 613-624. https://doi.org/10.1016/j.ymssp.2015.09.023.

[9] L. Rubio, J. Fernández-Sáez, and A. Morassi. The full nonlinear crack detection problem in uniform vibrating rods. Journal of Sound and Vibration, 339, (2015), pp. 99-111. https://doi.org/10.1016/j.jsv.2014.11.011.

[10] L. Rubio, J. Fernández-Sáez, and A. Morassi. Crack identification in non-uniform rods by two frequency data. International Journal of Solids and Structures, 75, (2015), pp. 61-80. https://doi.org/10.1016/j.ijsolstr.2015.08.001.

[11] G. M. L. Gladwell and A. Morassi. Estimating damage in a rod from changes in node positions. Inverse Problems in Engineering, 7, (3), (1999), pp. 215-233. https://doi.org/10.1080/174159799088027695.

[12] N. T. Khiem, L. K. Toan, and N. T. L. Khue. Change in mode shape nodes of multiple cracked bar: I. The theoretical study. Vietnam Journal of Mechanics, 35, (3), (2013), pp. 175188. https://doi.org/10.15625/0866-7136/35/3/2486.

[13] N. T. Khiem, L. K. Toan, and N. T. L. Khue. Change in mode shape nodes of multiple cracked bar: II. The numerical analysis. Vietnam Journal of Mechanics, 35, (4), (2013), pp. 299-311. https://doi.org/10.15625/0866-7136/35/4/2487.

[14] N. T. Khiem, P. T. Hang, and L. K. Toan. Crack detection in pile by measurements of frequency response function. Nondestructive Testing and Evaluation, 31, (2), (2016), pp. 122-141. https://doi.org/10.1080/10589759.2015.1081904.

[15] M. Dilena and A. Morassi. Structural health monitoring of rods based on natural frequency and antiresonant frequency measurements. Structural Health Monitoring, 8, (2), (2009), pp. 149-173. https://doi.org/10.1177/1475921708102103.

[16] M. Dilena and A. Morassi. Reconstruction method for damage detection in beams based on natural frequency and antiresonant frequency measurements. Journal of Engineering Mechanics, 136, (3), (2010), pp. 329-344. https://doi.org/10.1061/(asce)0733-9399(2010)136:3(329).

[17] L. Rubio, J. Fernández-Sáez, and A. Morassi. Identification of two cracks in a rod by minimal resonant and antiresonant frequency data. Mechanical Systems and Signal Processing, 60, (2015), pp. 1-13. https://doi.org/10.1016/j.ymssp.2015.01.025.

[18] T. G. Chondros, A. D. Dimarogonas, and J. Yao. Longitudinal vibration of a continuous cracked bar. Engineering Fracture Mechanics, 61, (5-6), (1998), pp. 593-606. https://doi.org/10.1016/s0013-7944(98)00071-x. 